\title{
Cecum Dieulafoy's Lesion Presenting with Recurrent Lower Gastrointestinal Bleeding: Case Report
}

\author{
Ludmila Resende Guedes, Silas Castro de Carvalho, Vitor Nunes Arantes, Arthur Manoel Braga de Albuquerque \\ Gomes, Daniel Antônio de Albuquerque Terra, Nathália da Silva Braga and Nicoly Eudes da Silva Dias \\ Endoscopy Unit of Alfa Institute of Gastroenterology at Hospital das Clínicas da Universidade Federal de Minas Gerais (UFMG), \\ Belo Horizonte, MG 30130100, Brazil
}

\begin{abstract}
Introduction: Gastrointestinal Dieulafoy's lesion is a rare entity, of unknown etiology, and corresponds to an arterial malformation at the submucosal space that can be a source of life-threatening hemorrhage. We report a case of a Cecum Dieulafoy's bleeding lesion that was managed endoscopically with a favorable outcome. Case report: Female, 70-year-old, diagnosed with type 2 diabetes, hypothyroidism, and chronic heart failure associated with rheumatic mitral stenosis, submitted to biological valve replacement in 2006 and with permanent atrial fibrillation using vitamin K antagonist anticoagulant for thrombosis prophylaxis. Her background includes a stroke in 2004 without any permanent disability. In 2016, the patient experienced voluptuous and painless lower gastrointestinal bleeding with severe acute anemia, requiring hospital admission, fluid resuscitation and blood transfusion. Urgent colonoscopy revealed a small reddish vascular malformation at the cecum with oozing active bleeding, about 3 mm in size. Initially argon plasma coagulation was performed with satisfactory and immediate hemostasis. One week later, she recurred with hematochezia. The lesion at the cecum was reassessed and it was possible to notice a large caliber vessel surrounded by a normal appearance mucosa, compatible with Dieulafoy's lesion and it was treated with an endoscopic clip placement with a good long-term response. Conclusion: Endoscopy is the method of choice for diagnosis of Dieulafoy's lesion and may provide efficient treatment with mechanical hemostasis such as endoclip placement with a high success rate.
\end{abstract}

Key words: Dieulafoy's lesion, lower gastrointestinal bleeding, endoscopy, endoscopic clip.

\section{Introduction}

Dieulafoy’s lesion is a rare entity, of unknown etiology, which corresponds to an arterial malformation at the submucosal space that can eventually be a source of bleeding. The most common location is the proximal gastrointestinal tract, especially the stomach, however it can also be found in other organs, such as the bronchial tree [1]. Preferentially, its occurrence is observed in elderly and male patients, with a male: female ratio of 2:1; often hospitalized; users of either NSAIDs (nonsteroidal anti-inflammatory drugs), aspirin or warfarin; with known comorbidities, especially cardiopulmonary dysfunction or chronic kidney disease and without a past history of gastrointestinal pathology [2]. The

Corresponding author: Ludmila Resende Guedes, M.D., research field: endoscopy and gastroenterology. presentation usually is either a life-threatening massive hemorrhage or recurrent bleeding with origin source frequently overlooked at index endoscopy. Consequently, misdiagnosis and delayed treatment lead to a mortality rate from Dieulafoy bleeding two-fold higher comparing to other etiologies of gastrointestinal hemorrhage [3]. Endoscopy performed by an experienced operator is the method of choice for diagnosis and when the lesion is properly identified, endoscopic treatment can achieve a high success rate, which ranges from 70 to $98 \%[1,4,5]$.

\section{Case Report}

We report a clinical case experienced by the endoscopic team of our institution. Female, 70 years old, diagnosed with type 2 diabetes, hypothyroidism, chronic heart failure associated with rheumatic mitral stenosis, submitted to biological valve replacement in 
2006 and with permanent atrial fibrillation using vitamin $\mathrm{K}$ antagonist anticoagulant (warfarin) for thrombosis prophylaxis. Her background includes a stroke in 2004 without any permanent disability. In 2016, the patient experienced voluptuous and painless lower gastrointestinal bleeding with severe acute anemia, requiring hospital admission, fluid resuscitation and blood transfusion. Since the patient had a high thromboembolic risk in a scenario of hemodynamic stability and apparent spontaneous interruption of hemorrhage, an elective colonoscopy was scheduled after bridging therapy with low weight molecular heparin. Colonoscopy revealed a small reddish vascular malformation at the cecum with oozing active bleeding, about $3 \mathrm{~mm}$ in size. Initially argon plasma coagulation was attempted with immediate hemostasis. Nevertheless, one week later, she recurred with hematochezia. The lesion at the cecum was reevaluated with a repeated colonoscopy and it was possible to notice a large caliber vessel surrounded by a normal appearance mucosa, suggestive of Dieulafoy's lesion (Fig. 1). An endoscopic clip was successfully placed at bleeding vessel (Fig. 2). The patient has remained without further bleeding episodes for one-year follow-up.

\section{Discussion}

In 1884, Gallard first described gastric vascular lesions as "milliary aneurisms". However, these lesions were named after George Dieulafoy, who was a French surgeon that detailed the malformation anatomy and published in 1898 three cases of young men that presented fatal hemorrhage due to a new condition named "exulceratio simplex" which was thought, at that time, to correspond to the first stage of peptic ulcer $[2,6]$.

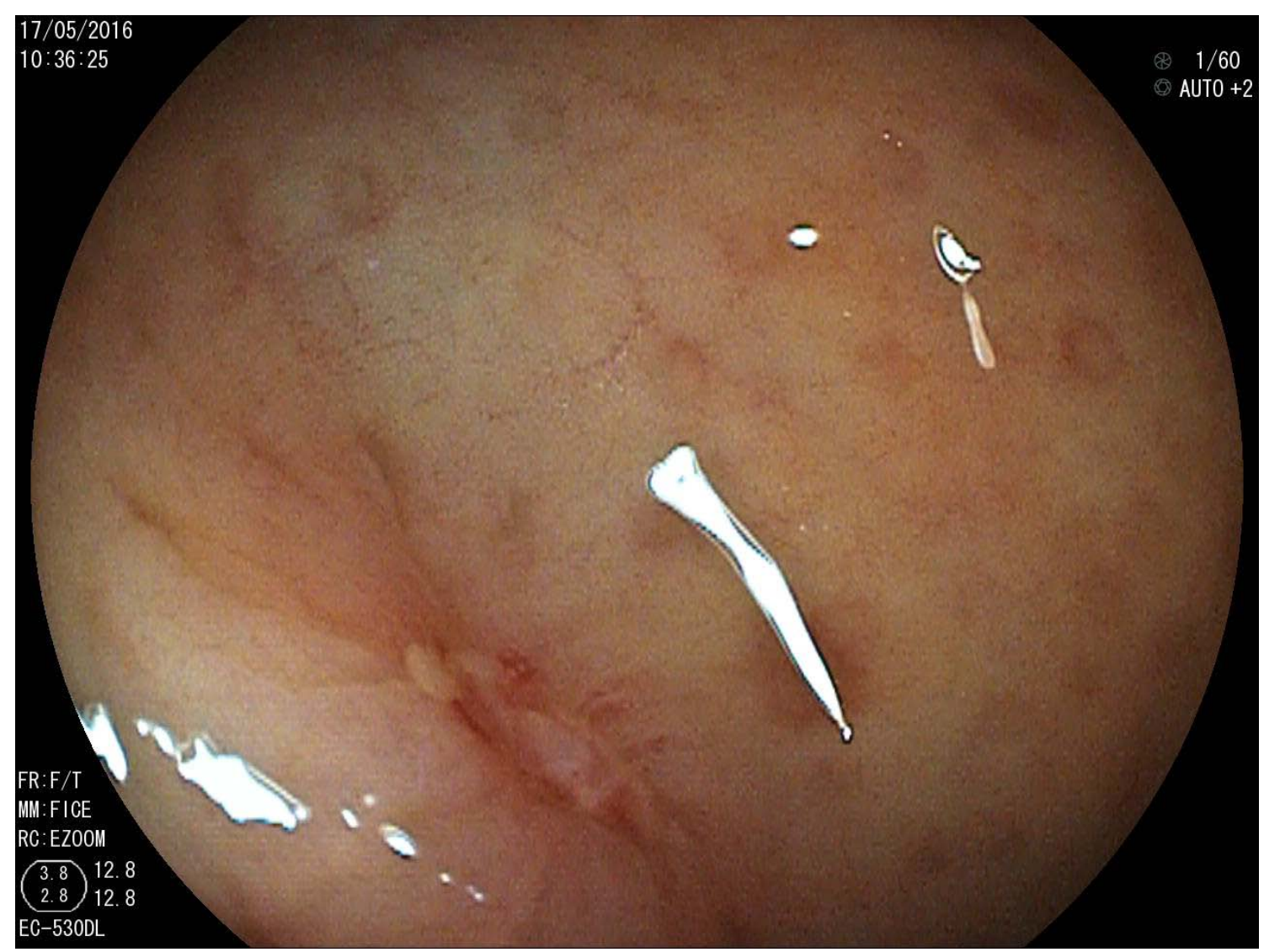

Fig. 1 Dieulafoy's lesion at the cecum. 


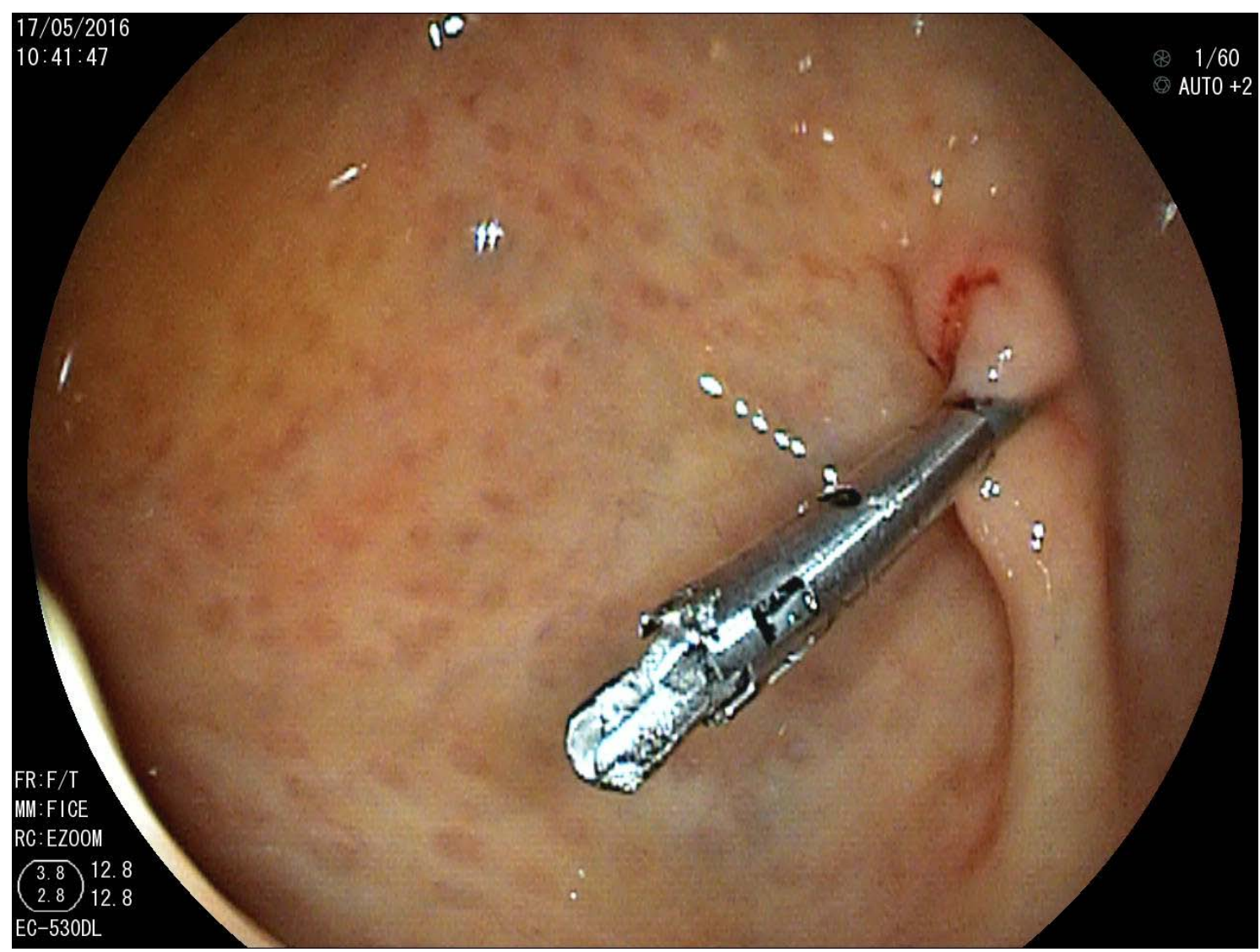

Fig. 2 Endoscopic clip placed at the lesion.

The pathogenesis is determined by an absence of ramification of an arterial submucosal vessel, originating an ectasic and superficial artery that is prone to laceration and consequently bleeding. The exact trigger of arterial rupture and bleeding is uncertain, but colonic trauma due to passage of solid fecal material is a possible mechanism [4]. About 1-2\% of gastrointestinal bleeding is attributed to these malformation, although the real prevalence is unknown since it can be asymptomatic [7].

The most common location of Dieulafoy's lesion is the stomach (71\%), especially along the lesser curvature of the gastric body, within $6-10 \mathrm{~cm}$ of the gastro esophageal junction, followed by the duodenum (15\%) and esophagus (8\%), whereas the colon accounts for only $2 \%$ of the cases [1, 4, 2]. Although even more rare, other organs involvement such as the bronchial tree have been also reported, with a total of 12 cases described [8].
Chronic alcohol drinking and antiplatelet drugs intake are possible for intervening factors that may contribute to Dieulafoy's lesion formation, with an Odds Ratio of 3,9 (1,6-9,5 CI 95\%) and 2,8 (1,2-6,2 CI $95 \%)$, respectively [5]. It is of note that hypertensive patients may experience high morbidity and mortality rates [9].

Endoscopy is the key to both diagnosis and treatment and it has enabled a dramatic decrease in mortality rates (from $80,0 \%$ to $8,6 \%$ ) in patients with hemorrhage due to Dieulafoy's lesion [2]. Nevertheless, the presentation is variable, hence the correct identification can still be difficult and up to $33 \%$ of lesions might be overlooked in the index endoscopy requiring more than one procedure to achieve a proper diagnosis and treatment as observed in this case $[7,10]$. Angiography can be useful to identify the location of bleeding, especially in those cases not identified by esophagogastroduodenoscopy [11]. 
However the performance of endovascular treatment has not shown promising results [11].

The endoscopic treatment involves sclerosants injection, such as epinephrine, alcohol, cyanoacrylate, hypertonic glucose; thermic mechanisms like argon plasma coagulation or mechanic hemostasis with endoscopic clips or band ligation, or even a combination of different hemostatic methods [11-13].

There is scarce data that compared the efficacy between the different hemostatic methods and the results are conflicting [14]. Nonetheless, some recent reports have suggested that mechanic hemostasis with band ligation and hemoclips are the first line endoscopic treatment, as demonstrated in this case [3, 4, $15,16]$. The overall risk of rebleeding ranges from 9 to $40 \%$ and kidney disease, infection, arterial spurting bleeding and monotherapy treatment with epinephrine are known predisposing factors $[4,13,17]$.

To date there are insufficient data to recommend proton pump inhibitors or other acid suppressive medications are as adjuvant therapy for gastric Dieulafoy's bleeding [11].

When conventional endoscopic therapy fails, endoscopic ultrasound guided technics can be an alternative approach in expert centers [18]. Surgery is a rescue option, indicated after unsuccessful or failed endoscopic and angiographic interventions. Intraoperative endoscopy can be performed to assist surgeons localizing the bleeding site [11].

Dieulafoy's lesion, although rare, has a big impact in morbidity and mortality associated with gastrointestinal bleeding. Therefore, it is a relevant issue in the research field and by reporting this case we not only add data to the literature for further investigation with regard to diagnosis and treatment but also we stimulate endoscopists to consider this entity among the differential diagnoses when performing an endoscopy in propaedeutic of bleeding.

\section{Conclusions}

Dieulafoy's lesion at the cecum is an extremely uncommon source of lower gastrointestinal bleeding. The diagnosis can be challenging and depends on the endoscopist expertise and a high suspicious rate particularly in patients with massive bleeding after a failed index endoscopy. Currently mechanic endoscopic hemostasis with endoclips or band ligation is the therapy of choice.

\section{References}

[1] Lara, L. F., Sreenarasimhaiah, J., Tang, S., Afonso, B. B., and Rockey, D. C. 2010. "Dieulafoy Lesions of the GI Tract: Localization and Therapeutic Outcomes.” Dig. Dis. Sci. 55 (12): 3436-41.

[2] Baxter, M., and Aly, E. H. 2010. “Dieulafoy’s Lesion: Current Trends in Diagnosis and Management.” Ann. $R$ Coll. Surg. Engl. 92 (7): 548-54.

[3] Patel, P., and Tobi, M. 2011. "Dieulafoy-Like Lesion Bleeding: In the Loop.” Gastroenterol Hepatol (N Y) 7 (4): 271-3.

[4] Jeon, H. K., and Kim, G. H. 2015. "Endoscopic Management of Dieulafoy’s Lesion.” Clin. Endosc. 48 (2): 112-20.

[5] Shin, H. J., Ju, J. S., Kim, K. D., Kim, S. W., Kang, S. H., Kang, S. H., et al. 2015. "Risk Factors for Dieulafoy Lesions in the Upper Gastrointestinal Tract.” Clin Endosc.

[6] Karamanou, M., Fiska, A., Demetriou, T., and Androutsos, G. 2011. "Georges-Paul Dieulafoy (1839-1911) and the First Description of Exulceratio Simplex." Ann. Gastroenterol 24: 188-91.

[7] Raimondo, M., Esmadi, M., Fisher, K., and Hewlett, A. 2017. "Atypical Appearance of a Rectal Dieulafoy Lesion Commentary.” Gastrointest Endosc 1.

[8] Wadji, M. B., and Farahzadi, A. 2017. "Dieulafoy's Disease of the Bronchial Tree: A Case Report.” Sao Paulo Med. J. 135 (1354).

[9] Kamal, M., Santhanam, P., and Rayyan, Y. M. 2016. "Hypertension and Clinical Outcomes of Patients With Gastrointestinal Submucosal Vascular (Dieulafoy) Lesional Hemorrhage.” J. Clin. Hypertens 18 (7): 710.

[10] Saleh, R., Lucerna, A., Espinosa, J., and Scali, V. 2016. "Dieulafoy Lesion: The Little Known Sleeping Giant of Gastrointestinal Bleeds.” Am. J. Emerg. Med. 34: 2464.e3-5.

[11] Lee, Y. T., Walmsley, R. S., Leong, R. W. L., and Sung, J. J. Y. 2003. “Dieulafoy’s Lesion.” Gastrointest Endosc 58 (2): 236-43.

[12] Benatta, M. A., and Grimaud, J. C. 2017. "Band Ligation for a Gastroesophageal Junction Dieulafoy’s Lesion.” Pan. Afr. Med. J. 26: 181. 
[13] Jamanca-Poma, Y., Velasco-Guardado, A., Piñero-P, C., Calder-Begazo, R, Umaña-Mej, J., Geijo-Mart, F., et al. 2012. "Prognostic Factors for Recurrence of Gastrointestinal Bleeding Due to Dieulafoy’s Lesion.” World J. Gastroenterol 18 (40): 5734.

[14] Kasapidis, P., Georgopoulos, P., Delis, V., Balatsos, V., Konstantinidis, A., and Skandalis, N. 2002. "Endoscopic Management and Long-Term Follow-up of Dieulafoy's Lesions in the Upper GI Tract.” Gastrointest Endosc 55 (4): 527-31.

[15] Chung, I. K., Kim, E. J., Lee, M. S., Kim, H. S., Park, S. H., Lee, M. H., et al. 2000. "Bleeding Dieulafoy's Lesions and the Choice of Endoscopic Method: Comparing the Hemostatic Efficacy of Mechanical and Injection
Methods.” Gastrointest Endosc 52 (6): 721-4.

[16] Yamaguchi, Y., Yamato, T., Katsumi, N., Imao, Y., Aoki, K., Morita, Y., et al. 2003. "Short-Term and Long-Term Benefits of Endoscopic Hemoclip Application for Dieulafoy's Lesion in the Upper GI Tract.” Gastrointest Endosc 57 (6): 653-6.

[17] Park, S-H., Lee, D-H., Park, C-H., Jeon, J., Lee, H-J., Lim, S-U., et al. 2015. "Predictors of Rebleeding in Upper Gastrointestinal Dieulafoy Lesions.” Clin. Endosc 48: 385-91.

[18] Hall, P. S. J., Teshima, C., May, G. R., and Mosko, J. D. 2017. "Endoscopic Ultrasound-Guided Vascular Therapy: The Present and the Future." EUS-Guided Ther Interv. Clin. Endosc 50: 138-42. 